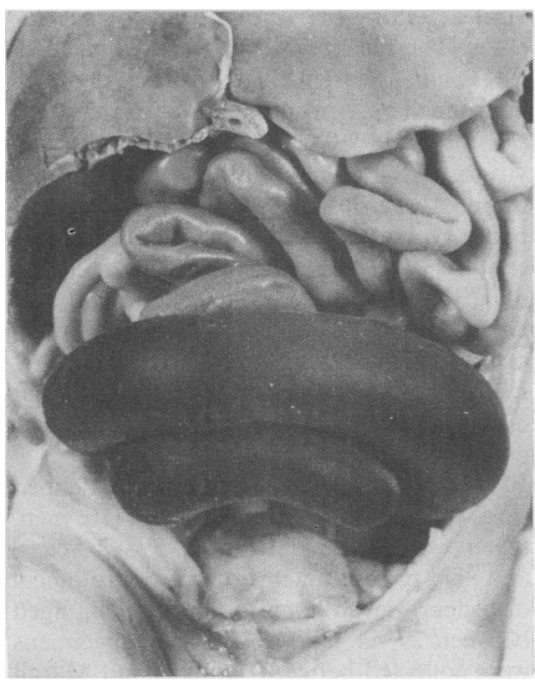

Fig 5 Volvulus of lower ileum.

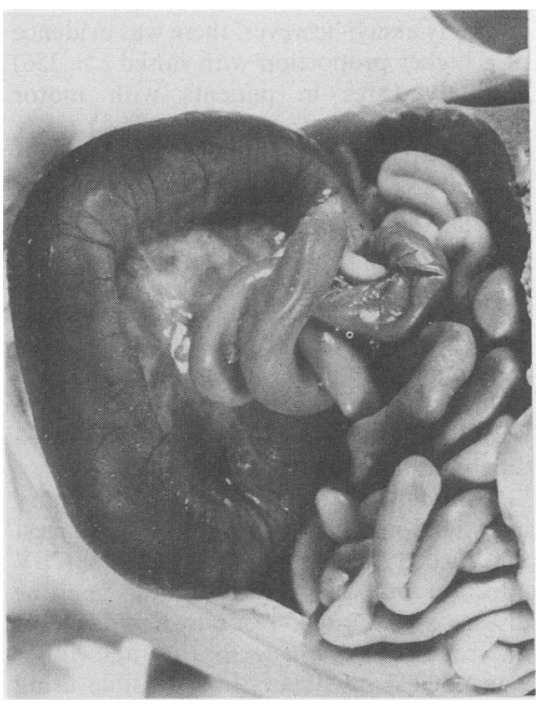

Fig 6 Same case, volvulus displayed to show complicated torsion.

not show gross or histological evidence of infarction and were distended with meconium. The caecum in all cases was normally tethered to the posterior abdominal wall and no evidence of malrotation or congenital bands was seen. In one fetus (case 1) there was adherence by loose adhesions of distended bowel to the liver and there was a small amount of bleeding into the abdominal cavity with a subcapsular haematoma in the fetus of case 3 . The bowel above the volvulus was not overtly distended and the distal small and large bowel were collapsed. In case 1 the placenta was received fragmented, having been evacuated after delivery and it showed pronounced chorioamnionitis. In case 4 there was a mild degree of chorioamnionitis but in the other two cases placentas and membranes were normal. The pancreas together with other internal organs was examined histologically in the fetuses of cases 2 and 4 and these showed normal appearances.

Two issues are posed by these intrauterine abdominal catastrophies - namely, the cause of the volvulus and the reason for the premature labour.

No congenital abnormality of the bowel was shown although the possibility of an abnormal mesentery could be a factor. I am not aware of any way of measuring such a condition, especially as the distended loops of the volvulus would themselves change the mesentery after the event. It is more likely that in these fetuses the arrival of meconium, which is being formed in increasing amounts during intrauterine growth, for some reason acts as a bolus in a dependent loop or loops of ileum and peristalsis completes volvulus formation. The fact that all the fetuses were between 20 and 22 weeks would, I suggest, support this hypothesis. There was no suggestion in any of the family pedigrees that volvulus could have been an unusual presentation of mucoviscoidosis and in two cases the pancreas was normal.

It is perhaps important that, although there was gross evidence of ischaemia, none of the cases had actual infarction of the bowel. It would seem logical to assume that the development of a volvulus would affect the fetus in a similar manner to that same stimulus in life after birth and that the "pain" and haemodynamic consequences following distension and stasis of a segment of bowel would stimulate the fetal adrenal glands. Although the role of the fetus in the initiation of labour is still contentious, it is thought that the adrenal may precipitate the train of events that induces labour. All the fetuses were "fresh" and in one case the fetal heart had been recorded up to two hours before delivery. My intuition leads me to believe that the cause (volvulus) had led to the effect (miscarriage).

TG ASHWORTH

Department of Morbid Anatomy and Histopathology,

Walsgrave Hospital, Coventry.

\section{Increased isolation of anaerobes at low cost}

Few would disagree that the isolation rate of anaerobes from clinical specimens can be increased by using selective media or prolonged incubation. Selective anaerobic media are used by many laboratories but prolonged incubation of plates beyond two days is usually reserved for those specimens in which slow growing anaerobes are suspected-a major pathogen in a subphrenic abscess. The procedure is not usually thought to be cost effective for routine specimens.

Over four months 1558 routine pus swabs were plated on to routine and selective media and incubated anaerobically for five days. The selective media used were WilkinsChalgren anaerobe agar (Oxoid) with 5\% horse blood supplemented with Gram negative anaerobe additive (Oxoid) or with non-sporing anaerobe supplement (Oxoid) and $0.1 \% \mathrm{v} / \mathrm{v}$ Tween 80 . Specimens were surface spread on to half plates, and a $5 \mu \mathrm{g}$ metronidazole disc placed at the interface of the innocula and the plates incubated anaerobically at $37^{\circ} \mathrm{C}$ for 48 hours. After examination for a zone around the metronidazole disc to indicate the presence of anaerobes all plates were reincubated for a further three days.

Anaerobes were isolated from 158 specimens $(10 \cdot 1 \%)$ after 48 hours and a further $98(6 \cdot 3 \%)$ after further incubation for a total of five days.

The sites from which anaerobes were isolated only after prolonged incubation fell within the following broad categories: ulcers/skin ( $n=60$ or $61 \%$ ), postoperative specimens $(n=12$ or $12 \%)$, "orthopaedic" specimens $(n=9$ or $9 \%)$, ear swabs $(n=9$ or $9 \%$ ), abscess material $(n=6$ or $6 \%$ ), swabs from gangrenous area $(n=2$ or $2 \%)$. These results show that prolonged anaerobic incubation of pus specimens increases the isolation rate of anaerobes.

I recognise that metronidazole resistant anaerobes would not be detected by the suggested method. Placing a $50 \mu \mathrm{g}$ gentamicin disc at the interface of the surface spreads would overcome this problem but is more time consuming, as all organisms growing up to the disc need further investigation. Clostridia will fail to grow on the suggested media but it was felt that it would be unrealistic to introduce further selective or non-selective media as blood agar was used routinely. It was noted that Bacteroides ureolyticus, which is reported to be inhibited by selective agents present in the supplements, was occasionally isolated on the selective media. 
The procedure described is inexpensive, relatively fast, and increases the isolation rate of anaerobes, most of which are found in ulcers. A study is proposed to evaluate the clinical importance of these findings.

JANET GOODLAND Public Health Laboratory, Musgrove Park Hospital, Taunton, Somerset. TAI 5DB.

\section{Unexpected expectoration}

We would like to draw attention to the unusual appearance of a bronchial cast that first led us to believe a patient had expectorated a portion of lung during the course of a respiratory arrest, (figure).

A 58 year old man was admitted with acute chest pain and had known chronic obstructive airways and coronary artery diseases. Shortly after admission he had a respiratory arrest and during resuscitation a length of reddish brown tissue $14 \mathrm{~cm} \times 1.5 \mathrm{~cm} \times 1 \mathrm{~cm}$ was sucked from the back of the throat. It had the consistency and appearance of lung (fig la). Microscopical examination showed irregular air spaces with thin walls resembling alveoli (fig lb). The walls comprised red cells, eosinophils, and acellular eosinophilic strands. To ensure that this was not lung tissue, a reticulin stain was necessary. This confirmed the absence of any permanent structures of the alveolar wall.

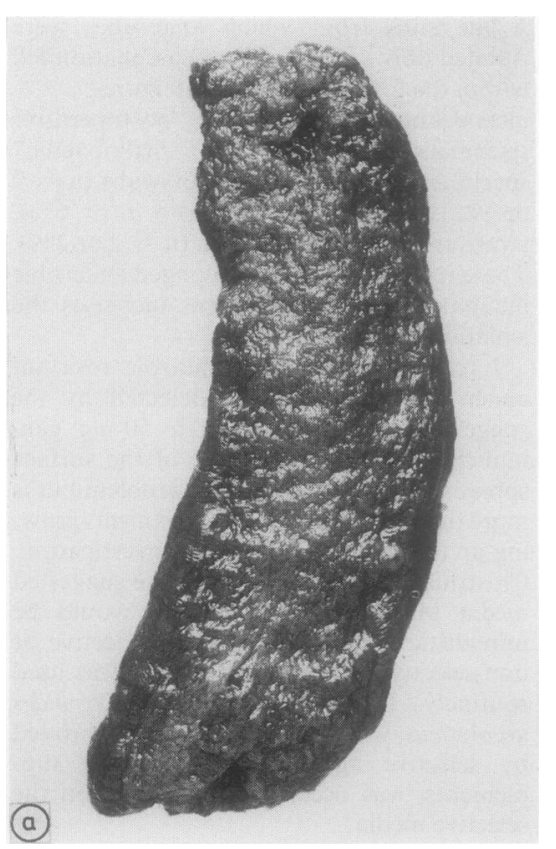

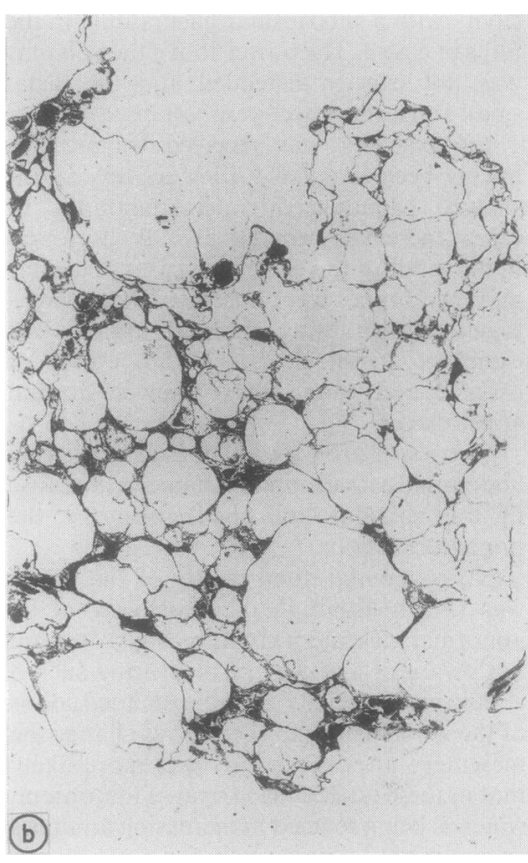

We were relieved to realise this was not lung and concluded that this was a bronchial cast "whisked" into its present "pulmonary" form by coughing. It clearly differs from the common descriptions of bronchial casts that show alternating cellular and acellular areas. The former exhibit large numbers of eosinophils, the latter are mostly mucous. CharcotLeyden crystals and Kurschmann's spirals are also common in the typical cast. Only one area was eventually found on the current case with laminated mucus strands that bore any resemblance to a typical bronchial cast.

M SUNDARESAN, AB PRICE, Department of Pathology, Northwick Park Hospital, Watford Road, Harrow, Middlesex

Non-polio enteroviruses and motor neurone disease

There is considerable interest in the possible

Table Results of Coxsackie B tests in patients and controls

\begin{tabular}{|c|c|c|c|c|c|}
\hline \multirow[b]{2}{*}{ Group } & \multirow[b]{2}{*}{ No $(\%)$ tested } & \multirow{2}{*}{$\begin{array}{l}\text { C B IgM } \\
\text { positive }\end{array}$} & \multicolumn{3}{|c|}{$C B$ neutralising antibody titres } \\
\hline & & & $\geqslant 512$ & 256 & $\geqslant 256$ \\
\hline $\begin{array}{l}\text { Patients } \\
\text { Controls* }\end{array}$ & $\begin{array}{l}17(100) \\
11(100)\end{array}$ & $\begin{array}{l}4(23 \cdot 5) \\
2(18)\end{array}$ & $\begin{array}{l}5(29) \\
1(10)\end{array}$ & $\begin{array}{l}7(41) \\
1(10)\end{array}$ & $\begin{array}{r}12(71) \\
2(20)\end{array}$ \\
\hline
\end{tabular}

viral aetiology of various neurological diø eases. The recent observation by one of (SK) of an apparent cluster of cases of mot neurone disease prompted us to examine the possible role of the group B Coxsacki viruses (CBV). The putative role of $\mathrm{CBV} \dot{\vec{m}}$ the post-viral fatigue syndrome is currenty being evaluated by us using the $\mu$-antiboo. capture ELISA technique for the detection of CBV IgM antibody.' Detection of the antibody implies recent or persisting infecr. tion. It is well established that this CBQ ELISA test can cross react with othẹ non-polio enteroviruses, ${ }^{23}$ thus extending its diagnostic scope.

We studied sera from 17 clinically defined cases of motor neurone disease $(10$ mew, seven women, aged between 39-87 yearif and 11 patients with other neurologicast disorders (seven men, four women, aged between 29-64 years). All serum specimeks were collected between January and March 1986 (table). There was little difference in the number of patients with motor neuron disease giving a CBV IgM positive respon compared with the control group. Using the more conventional CBV neutralising antibody assay, however, there was evidenes of a higher proportion with raised $(\geqslant 256)$ antibody titres in patients with motor neurone disease.

In evaluating these data it should pointed out that CBV was epidemic in the local community during 1985-86; this reflected by higher background titres asymptomatic adults assessed by both the CBV IgM and neutralising antibody assays. This may possibly explain the higher titre seen in our cases, but the number of patients studied was small, and the more sensitive CBV IgM assay failed to show a pronounced difference in the two groups studied.

Although the results of this preliminaty investigation are inconclusive, we suggegit that further epidemiological studies of $\mathrm{CB}$ infection in patients with motor neuroße disease are merited. Definitive evidence of the role of any virus in this and other neurological diseases is now feasible using the molecular biological techniques of in situ and Southern blot hybridisation to detects viral nucleic acid sequences in affectes
neurological tissues.

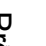

